\title{
CHARACTERIZATION OF DRY AGGREGATES IN CARBON ANODES BY IMAGE ANALYSIS
}

\author{
Dipankar Bhattacharyay ${ }^{1}$, Duygu Kocaefe ${ }^{1}$, Yasar Kocaefe ${ }^{1}$, Arunima Sarkar ${ }^{1}$, Brigitte Morais ${ }^{2}$, Jerome Chabot $^{2}$ \\ ${ }^{1}$ UQAC/Aluminerie Alouette Research Chair on Carbon, University of Québec at Chicoutimi, \\ 555 Boulevard de l'Université, Chicoutimi, Québec, Canada G7H 2B1 \\ ${ }^{2}$ Aluminerie Alouette Inc.; 400, Chemin de la Pointe-Noire, Sept-Îles, Québec, Canada G4R 5M9
}

Keywords: Carbon anodes, image analysis, form factor, roundness, particle characteristics, butt, coke

\begin{abstract}
Carbon anode is one of the key components in the electrolytic production of primary aluminum. Anodes are mainly composed of dry aggregates such as calcined petroleum coke and recycled materials with pitch as the binder. Granulometry of the dry aggregates is important to obtain good physical, chemical, electrical, and mechanical anode properties. Sieving can indicate the size range of particles, but it does not reveal much information about the shape or nature of the individual particles. This article presents an image analysis technique to study not only the granulometry, but also the physical characteristics (e.g. aspect ratio, roundness, form factor, etc.) of each and every particle. The custom-made software for the image analysis can also separately identify the butt and coke particles based on different shape parameters. This technique could help track changes in granulometry at different stages of the anode production and consequently improve the quality.
\end{abstract}

\section{Introduction}

Carbon anodes for the production of primary aluminum are manufactured using coal tar pitch as binder with dry aggregates such as calcined fresh petroleum coke, recycled butts, and rejected anodes. The dry aggregates, mixed with pitch, are compacted in a vibro-compactor to form 'green anode'. During different stages of fabrication, the granulometry and the distribution of particles can change. Analysis of the granulometry and the distribution of different particles can help improve the quality of anodes.

It is a known fact that the granulometry of dry aggregates and their distribution in an anode are two of the key factors that control anode properties such as density, electrical resistivity and $\mathrm{CO}_{2}$ /air reactivities. Xie et al. [1] studied the effect of granulometry on anode properties. Vogt et al. [2] described that the granulometry of dry aggregates can influence the thermal shock behavior of an anode.

Usually the granulometry of coke particles are analyzed using sieves of different size ranges. Sieving of the dry aggregates can show the amount of particles in a particular size range; however, it does not give any detailed information as to the type and characteristics of the particle.

Little work has been done on studying the effect of particle shape characteristics on anode properties. Azari et al. [3] studied the effect of coke particle characteristics on compression behavior of an anode paste. A Nikon Ephiphot optical microscope was used to take the picture of coke particles; and using Clemex vision software, particle characteristics such as aspect ratio, compactness, roughness, and roundness were measured. The limitation of using optical microscope is that it is difficult to analyze samples with a large range of particle sizes.
This work focuses on the development of an image analysis technique that can be applied to dry aggregates to analyze the granulometry and shape characteristics of different particles. Pictures are taken using ordinary digital camera which eliminates the constraint on the maximum size for particles.

\section{Methodology}

\section{Sample preparation}

A measured amount of dry aggregates was placed on a piece of white paper. A light was placed below the paper at a certain distance. A line was drawn on the paper, and its length was measured as a reference value.

\section{Instrumentation}

Picture of the dry aggregates was taken using a Sony DSC H3 camera with the macro setting using a zoom of 1x.

\section{Image analysis}

The captured image was analyzed using a custom-made software developed with Visual Basic 6.0.

\section{Change of color}

The coke particles appear gray to black whereas the paper appears whiter than the particles. Therefore, in the first step, the picture was changed to gray scale. All the pixels in a picture are composed of 3 primary colors, namely red (R), green (G), and blue (B). In the gray tone picture, the $R, G$, and $B$ values of each pixel were replaced by $(R+G+B) / 3$. Then, a threshold for the gray tone value was selected such that all the pixels below that value represent paper whereas the remaining pixels represent coke particles. Thus, using the threshold, the gray tone picture was binarized, and the colors of the pixels corresponding to the paper and particles were changed to black and white, respectively.

\section{Calculation of the area of coke particles}

The area of coke particles were calculated using flood-fill algorithm. The flood-fill is a recursive program and uses a stack data structure to fill all the points adjacent to a pixel (node) having a particular color (target-color) by another color (replacementcolor). The algorithm is given as:

Flood-fill (node, target-color, replacement-color):

1. If the color of node is not equal to target-color, return

2. Set the color of node to replacement-color

3. Enter the nodes (push) neighboring the original node in a stack. 
4. Take out (pop) the last node and perform Flood-fill.

5. Continue till the stack becomes empty.

6. Return.

The number of times the target-color (white) is replaced by the replacement color denotes the area of the coke particle in pixel units.

\section{Identification of the boundaries of each particle}

During the application of flood-fill algorithm to determine the area, the boundaries of coke particles can be identified. A pixel will be at the boundary of a coke particle if at least one of its neighbors is a black pixel. If a particle is found to be on the boundary, the color of the pixel is changed to cyan and the coordinates of all the pixels corresponding to every particle are saved in a table. Each particle was also assigned a unique identification number.

\section{Calculation of different physical characteristics of a particle}

Perimeter: The number of cyan color pixels on the boundary of a particle gives the perimeter of the particle in pixel units.

Maximum length: The distances between different points on the boundary of a particle are measured using the formula to measure the distance $(\mathrm{d})$ between two points $(\mathrm{x} 1, \mathrm{y} 1)$ and $(\mathrm{x} 2, \mathrm{y} 2)$ given by Equation 1:

$$
d=\sqrt{(x 1-x 2)^{2}+(y 1-y 2)^{2}}
$$

The maximum of all the measured distances gives the maximum length of the particle and the coordinates of pixels corresponding to the maximum length.

Maximum width: The width of any size will be perpendicular to the maximum length. If the slope of the line corresponding to the maximum length is $\mathrm{m}$, then the line corresponding to the any width will have a slope of $-1 / \mathrm{m}$. Thus, the widths of all lines with a slope of $-1 / m$ (i.e., intersecting the maximum length at right angle) connecting the points on the boundary of a particle were determined. The maximum of all those widths is the maximum width of the particle.

Aspect ratio: It is defined as the ratio of maximum length to minimum width (measured perpendicular to the maximum length) of a particle [4]. All the widths of a given particle are determined as explained above in the Maximum Width section. Then the minimum of all those widths is the required minimum width of the particle. Thus, the maximum length divided by the minimum width gives the aspect ratio of a particle. The aspect ratio is most sensitive to elongation, but not sensitive to irregularities on the boundary.

Roundness: Roundness is defined as:

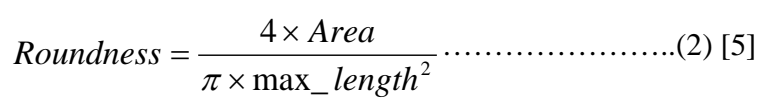

Using the values of area and maximum length, the roundness can easily be calculated. It gives an idea about the extent by which the edges and corners of a particle are rounded.
Root form factor: Root form factor is defined as:

$$
\text { Root }_{-} \text {form }- \text { factor }=\frac{2 \sqrt{\pi \times \text { Area }}}{\text { Perimeter }}
$$

Using the values of area and perimeter, form factor can easily be calculated. Form factor is sensitive to irregularities in the particle boundary [6].

\section{Identification of butts}

The butts can be distinguished from coke particles based on their physical appearance. Butts are usually large particles and have sharp corners. Unlike butt particles, coke particles are usually spherical or have rounded corners. Thus, butts can be described as particles having area higher than a threshold value having a form factor above a certain value.

\section{Calibration of a pixel with an actual length}

The length Lp of the line marked on the paper is measured in the same way described for the measurement of a maximum length in pixel units. If the measured length of the line is $\mathrm{La}$ in $\mathrm{mm}$, then each pixel represents a length of $\mathrm{La} / \mathrm{Lp} \mathrm{mm}$.

\section{Basis of identification of different size fractions}

While measuring the granulometry using sieves, a measured quantity of particles is taken in the topmost sieve which has the largest aperture. The other sieves are placed below that sieve with decreasing aperture. A pan is placed at the bottom. The assembly of the sieves is shaken in a mechanical shaker at a fixed amplitude and frequency. After a certain time, the shaker is stopped, and the weights of particles in different sieves are measured. During the shaking process, depending on their orientation, particles having a length or a width smaller than the aperture of a given sieve pass down to the following sieve with a smaller aperture. Thus, in this work, the size fractions are calculated based on maximum length as well as maximum width separately. Particles having a maximum length or a maximum width in a certain range are included in that particular size fraction.

\section{Calculation of the weight fraction for different size ranges by image analysis}

Assuming that the densities of the particles are the same, the weight fractions of the different size fractions are calculated. For similar density particles, the percentages of weight for different size fractions will be similar to the corresponding area percentages of the same size fractions. Thus, the sums of areas (in pixel units) of particles were calculated separately for different size fractions. Using these values, the area percentages for different size fractions are found, which in turn gives the weight fractions of the particles.

\section{Results and discussion}

A random mixture of dry aggregates was separated into a number of fractions using a shaker with sieve sizes of $8 \mathrm{~mm}, 6.3 \mathrm{~mm}, 4 \mathrm{~mm}$, and $2 \mathrm{~mm}$. Four fractions were taken to test the image analysis technique developed: $>8 \mathrm{~mm},-8 \mathrm{~mm}+6.3 \mathrm{~mm},-6.3 \mathrm{~mm}+4 \mathrm{~mm}$, $-4 \mathrm{~mm}+2 \mathrm{~mm}$; and they were remixed. The fraction $<2 \mathrm{~mm}$ (pan) was eliminated to avoid errors that could occur due to dust or fine 
particles. Then, the weight percentages of the four fractions considered were calculated based on their total weight. Table 1 shows the results of the granulometry calculated for the four fractions following the sieve analysis.

Table 1 Granulometry measured by sieve analysis

\begin{tabular}{|c|c|}
\hline Size fraction & Weight percentage \\
\hline$>8 \mathrm{~mm}$ & 18.449 \\
\hline$-8 \mathrm{~mm}+6.3 \mathrm{~mm}$ & 17.750 \\
\hline$-6.3 \mathrm{~mm}+4 \mathrm{~mm}$ & 29.310 \\
\hline$-4 \mathrm{~mm}+2 \mathrm{~mm}$ & 34.491 \\
\hline
\end{tabular}

As described above, the particles were spread on a white paper to take a picture. Figure 1 shows the picture of the particles placed on the white paper.

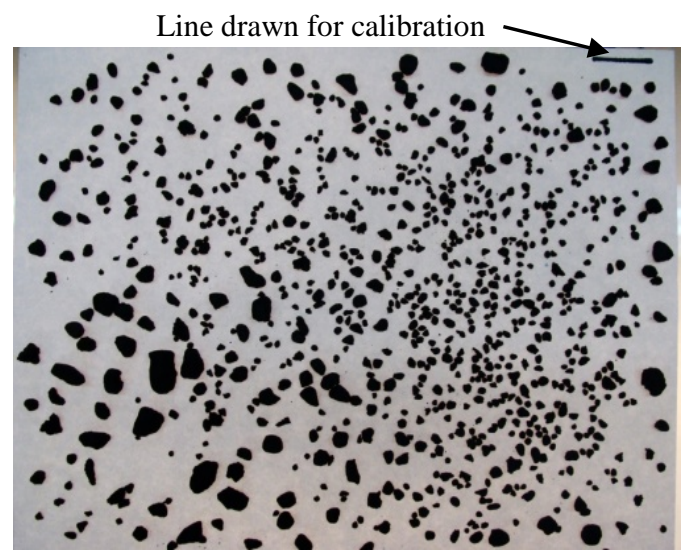

Figure 1. Picture of the dry aggregates

Then, the picture was binarized as shown in Figure 2 where the particles appear white and the paper appears black.

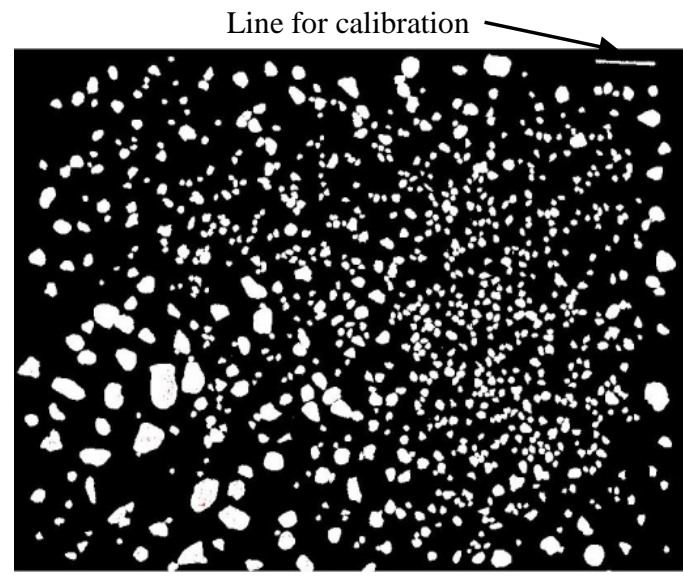

Figure 2. Binarized picture of the dry aggregates
The particle areas were measured by flood-fill algorithm, and the particle boundaries were determined by the image analysis technique developed. Figure 3 shows the processed picture where the particles, the particle boundaries, and the background (paper) are clearly seen.

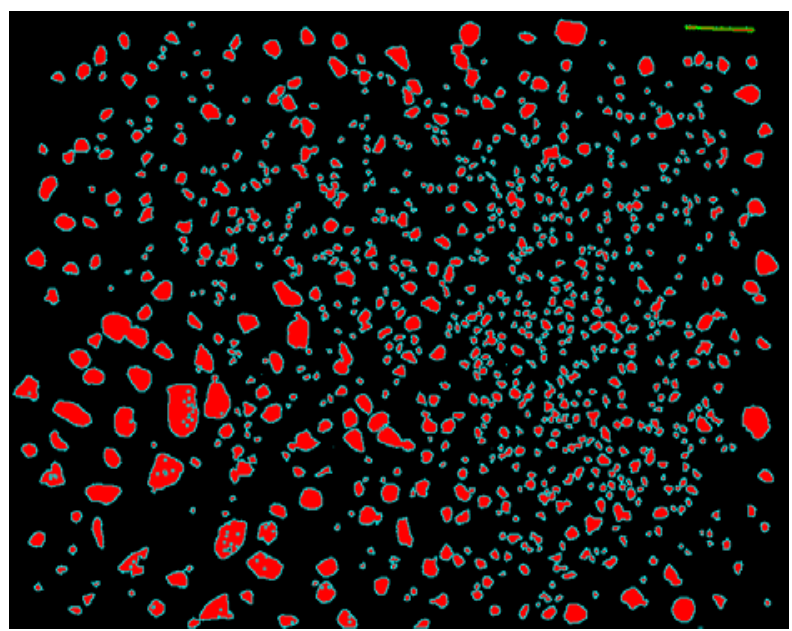

Figure 3. Processed picture of the dry aggregates

The pixel value was calibrated with the known length of the drawn line as explained previously. The calculated length and width of different particles were converted from pixel units to $\mathrm{mm}$ using the calibration value. Then, the sums of areas corresponding to different size fractions were calculated using the same limits ( $2 \mathrm{~mm}, 4 \mathrm{~mm}, 6.3 \mathrm{~mm}$, and $8 \mathrm{~mm})$. The values were then converted to weight percentage.

Table 2 shows the comparison of granulometry calculated by the image analysis technique (based on maximum length as well as maximum width) with the experimental values.

Table 2. Granulometry predicted by image analysis

\begin{tabular}{|c|c|c|c|}
\hline \multirow{2}{*}{ Size fraction } & \multicolumn{3}{|c|}{ Weight percentage } \\
\cline { 2 - 4 } & Experimental & $\begin{array}{c}\text { Based on } \\
\text { maximum } \\
\text { length }\end{array}$ & $\begin{array}{c}\text { Based on } \\
\text { maximum } \\
\text { width }\end{array}$ \\
\hline$>8 \mathrm{~mm}$ & 18.449 & 39.326 & 17.565 \\
\hline$-8 \mathrm{~mm}+6.3 \mathrm{~mm}$ & 17.750 & 14.843 & 16.391 \\
\hline$-6.3 \mathrm{~mm}+4 \mathrm{~mm}$ & 29.310 & 24.877 & 28.433 \\
\hline$-4 \mathrm{~mm}+2 \mathrm{~mm}$ & 34.491 & 20.862 & 33.299 \\
\hline$-2 \mathrm{~mm}+1 \mathrm{~mm}$ & - & 0.092 & 4.312 \\
\hline
\end{tabular}

Table 2 shows that the granulometry calculated based on the maximum width of the particles are much closer to the experimental values than those based on the maximum length. This shows that the maximum width is a better sieving indicator for a particle. If the maximum width of a particle is smaller than 
the opening of a sieve, the particle can pass through the sieve. The image analysis technique also identified some particles smaller than $2 \mathrm{~mm}$ which did not actually pass through the $2 \mathrm{~mm}$ sieve. This is quite likely due to their orientation or some hindrance by other particles.

To differentiate butt and coke particles, few randomly chosen butt and coke particles were analyzed. The particles are shown in Figure 4. The roundness, root form factor, aspect ratio as well as their product were calculated for each particle. The results are given in Table 3.

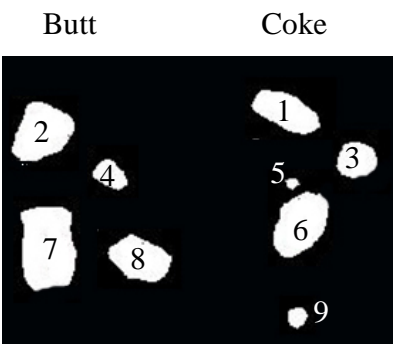

Figure 4. Butt and coke particles studied

Table 3 shows that the butt particles can be distinguished from coke particles based on the product of roundness, root form factor, and aspect ratio. It was observed that the products for butt particles are usually less than 0.7 . The products are usually higher in case of coke particles due to the surface roughness. The particles that appear spherical have high values of roundness as it seems to be the case for particles 3, 5, and 9 .

Table 3. Various shape factors for the particles shown in Figure 4

\begin{tabular}{|c|c|c|c|c|c|}
\hline Type & $\begin{array}{c}\text { Particle } \\
\text { ID }\end{array}$ & $\begin{array}{c}\text { Roundness } \\
\mathbf{( 1 )}\end{array}$ & $\begin{array}{c}\text { Root } \\
\text { form } \\
\text { factor } \\
\text { (2) }\end{array}$ & $\begin{array}{c}\text { Aspect } \\
\text { ratio } \\
\mathbf{( 3 )}\end{array}$ & $\begin{array}{c}\text { Product } \\
(\mathbf{1} \times \mathbf{2} \times \mathbf{3})\end{array}$ \\
\hline \multirow{4}{*}{ Butt } & 2 & 0.70 & 0.74 & 1.25 & 0.65 \\
\cline { 2 - 6 } & 4 & 0.67 & 0.72 & 1.37 & 0.66 \\
\cline { 2 - 6 } & 7 & 0.65 & 0.83 & 1.23 & 0.66 \\
\cline { 2 - 6 } & 8 & 0.64 & 0.73 & 1.45 & 0.68 \\
\hline \multirow{4}{*}{ Coke } & 1 & 0.49 & 0.72 & 2.17 & 0.77 \\
\cline { 2 - 6 } & 3 & 0.86 & 0.81 & 1.11 & 0.78 \\
\cline { 2 - 6 } & 5 & 1.00 & 0.88 & 0.99 & 0.91 \\
\cline { 2 - 6 } & 6 & 0.63 & 0.76 & 1.60 & 0.76 \\
\cline { 2 - 6 } & 9 & 0.87 & 0.84 & 1.18 & 0.86 \\
\hline
\end{tabular}

\section{Conclusions}

In this work, an image analysis technique has been described where pictures taken by an ordinary digital camera can be analyzed. The results show that the granulometry of dry aggregates can be calculated by this image analysis technique.

The technique can also identify different components in a mixture of dry aggregates based on characteristic shape factors of the components. In this study, the product of roundness, root form factor, and aspect ratio has been used to differentiate the butt and coke particles. This is indeed a simple approach. The reliability of the approach can be enhanced by incorporating a more advanced data analysis technique such as a perceptron-based artificial neural network. This image analysis technique can also be applied at certain steps of anode fabrication.

The fine particles (less than $2 \mathrm{~mm}$ in size) were excluded in the current analysis because such particles, especially dust, may cause handling difficulties. However, the work will continue to determine the limit for the particle size analysis (minimum particle size that can be handled by the image analysis technique).

\section{Acknowledgements}

The technical and financial support of Aluminerie Alouette Inc. as well as the financial support of the National Science and Engineering Research Council of Canada (NSERC), Développement économique Sept-Îles, the University of Québec at Chicoutimi (UQAC), and the Foundation of the University of Québec at Chicoutimi (FUQAC) are greatly appreciated.

\section{References}

[1] Y. Xie, D. Kocaefe, Y. Kocaefe, L. Wei, S. Zou, A. Wu, "Correlation between Anode Recipe and Anode", International Journal of Engineering and Innovative Technology, 2(8) (2013) 23-27.

[2] F. Vogt, R. Tonti, M. Hunt, L. Edwards, “A Preview of Anode Coke Quality in 2007”, Light Metals, (2004) 489-493.

[3] K. Azari, H. Ammar, H. Alamdari, D. Picard, M. Fafard, D. Ziegler, "Effects of Physical Properties of Anode Raw Materials on the Paste Compaction Behavior”, Light Metals, (2011), 11611164

[4] D. Gantenbein, J. Schoelkopf, G. P. Matthews, P.A.C. Gane, "Determining the size distribution-defined aspect ratio of rod-like particles” Applied Clay Science, 53 (2011) 538-543.

[5] A. Korolev, G. Isaac, "Roundness and Aspect Ratio of Particles in Ice Clouds”, Journal of the Atmospheric Sciences, 60, (2003), 1795-1808.

[6] L. Zhang, L.Tong, J. Gal, "Applied Mechanics, Progress and Applications", Proceedings of the Third Australasian Congress on Applied Mechanics, (2002) Sydney, Australia, World Scientific 